\title{
Research on Mechanism of Dead-time Influencing APF's Compensation Performance
}

\author{
J. Shen, X.L. Huang, X.H. Qu \\ School of Electrical Engineering, \\ Southeast University, \\ Nanjing, China
}

\begin{abstract}
At present, there are many methods to improve the compensation performance of active power filter (APF). However, to improve the reliability of APF, dead-time between two IGBT switches in the same bridge must be set large enough to guarantee the IGBT operating safely, which will impact the output performance of APF. This paper proposes a modeling method on a specific order harmonic to investigate the dead-time's influence to the compensation performance of APF. The theoretical analysis is well verified by the simulation results. At last, some methods are recommended to minimize the unexpected effect caused by dead-time.
\end{abstract}

Keywords-active power filter (APF); power quality; harmonic; dead-time effect

\section{INTRODUCTION}

With the rapid development of power electronic technology, more and more power electronic equipment are used in a variety of domains. However, the power electronic devices inject the harmonic current and reactive current to the power system which cannot ignore, lead the current and voltage waveform distortion. In addition, with the various kinds of digital electrical equipment and precision electronic instruments' wide use, the requirements of power quality in power system will be more and more strict and even harsh [1-4].

Compared to the passive power filter, active power filter (APF) has many advantages, such as dynamic compensation of harmonics elimination, reactive power, and fast dynamic response. APF has been developed greatly and has been widely used in power distribution network [5]. However, APF itself contains a lot of power electronic switching device; the access network is a special nonlinear load. The switching speed, control method, and the selected parameter are all the factors likely to affect the performance of APF harmonic compensation, make itself also produce a certain amount of harmonic, which cannot make up the nonlinear load harmonic to $100 \%$.

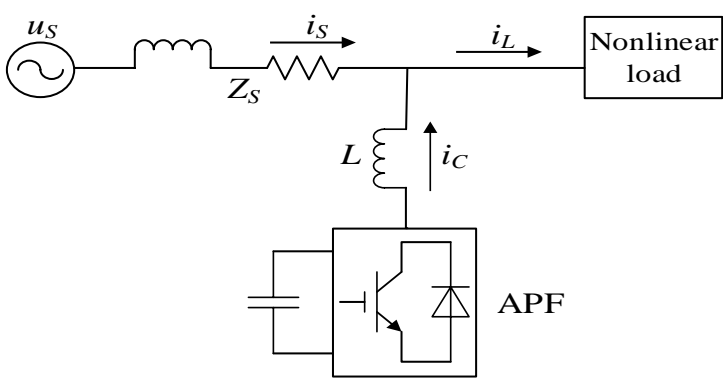

Figure 1. APF circuit structure.

This paper based on the APF electrical structure and operational principle, establish the simulation model of APF, analyze the causes of power switch device IGBT controlling dead zone, use a specific harmonic analysis method to control the dead zone, and set up model for the impact on APF compensation performance, quantitative analysis the mechanism of effect of the control delay on the APF compensation performance by mathematical reasoning. Finally verify the correctness of reasoning through simulation, and sum up some methods of reducing dead time effect.

\section{THE BASIC OPERATIONAL PRINCIPLE OF SHUNT APF}

The topology circuit structure of APF is as shown in Figure 1 [6]. In the figure, $u_{S}$ and $i_{S}$ are, respectively, the current and voltage for grid, $i_{L}$ is the harmonic current generated by nonlinear load, $i_{C}$ is the compensation current outputed by APF, $Z_{S}$ is the system equivalent impedance.

The basic operational principle of APF is: first, detect the grid voltage and the load harmonic current, get the output compensation current signal by reference current calculation method, and then through the current tracking control method make the APF output compensation current track the reference current signal, so that the APF output current can offset with harmonic components of load current.

Figure 2 is the equivalent shunt circuit of APF principle; shunt APF is equivalent to a controlled current source $i_{C}$, determine the APF output current according to the load harmonic current $i_{L}$, that is, $i_{C}=f\left(i_{L}\right)$.

Apparently, according to Kirchhoff's current law: 


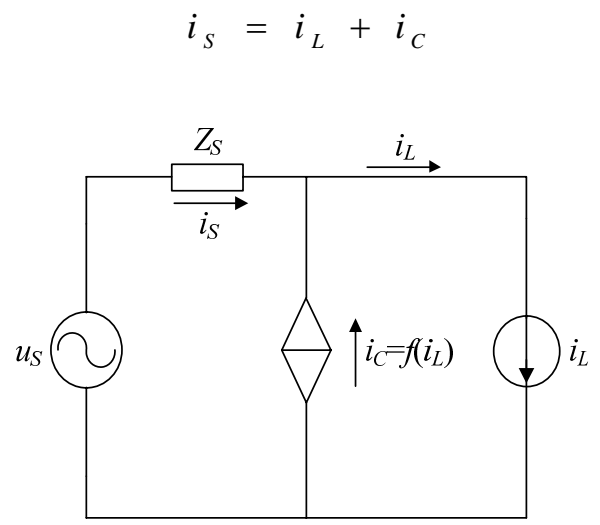

Figure 2. Equivalent circuit of APF compensation principle.

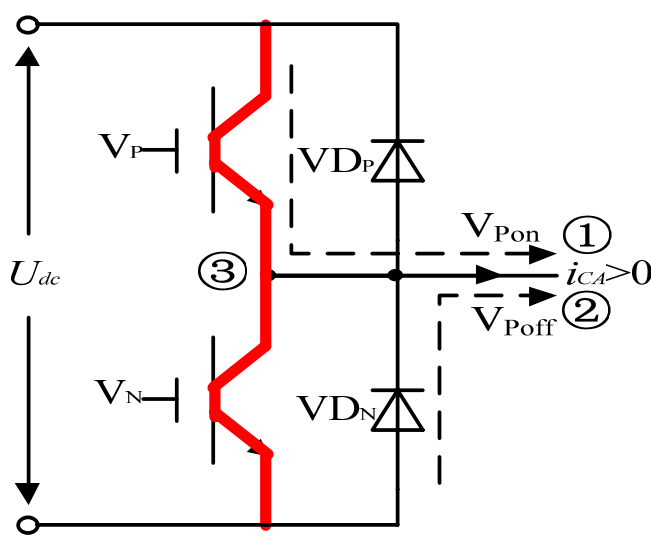

Figure 3. Single bridge arm APF compensating current path.

From Eq. (1), we can ensure that as long as control $i_{C}$ being equal to APF output compensation current harmonic components of nonlinear load current $i_{L h}$, the grid current $i_{S}$ will be no harmonic component, which can suppress the harmonic current.

\section{THE APF DEAD TIME CONTROL}

As shown in Figure 3, the current path and the path to the APF compensation current polarity [7] is positive, the normal path in different switching condition, the path to short-circuit path.

In order to avoid the APF main circuit path arise the short circuit fault, "dead time" is proposed to solve this problem, namely the main circuit of the APF IGBT bridge arm bring into a short time blocked when drive complementary control signals, blocked the drive signals in the same bridge arm to ensure a reliable IGBT being off, another IGBT is reliable conduction.

\section{SPECIFIC HARMONIC DEAD TIME EFFECT MODEL}

In order to prevent the main circuit of the APF bridge two arm power switching devices $V_{P}$ and $V_{N}$ from through short, the dead time is considered to join in the driving control signal. During the dead time, the same bridge arm two IGBT have no current flows. The current flows through the corresponding anti parallel diode $V D_{p}$ or $V D_{N}$ to maintain the continuity. Considering the dead time, the output voltage of APF is shown in Figure 4. In the figure, $V_{P}$ and $V_{N}$ represent complementary symmetry driving signal waveform in the same bridge arm circuit of the upper and lower two IGBT switch tube without dead zone, $V_{P}^{\prime}$ and $V_{N}$ 'represent the actual driving signal waveform with the dead time adding in, $u_{A B}$ and $u_{A B}$ 'APF respectively represent ideal output voltage waveform of the main circuit without dead zone and the practical output voltage waveform adding in the dead zone, $u_{t d}$ is the dead voltage waveform because of dead time effect, that is the difference of the actual output voltage caused by dead zone and the ideal output voltage.

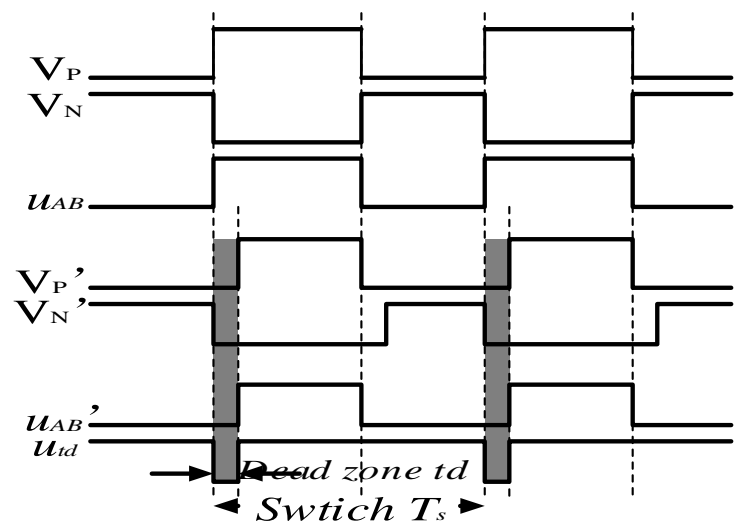

(a)

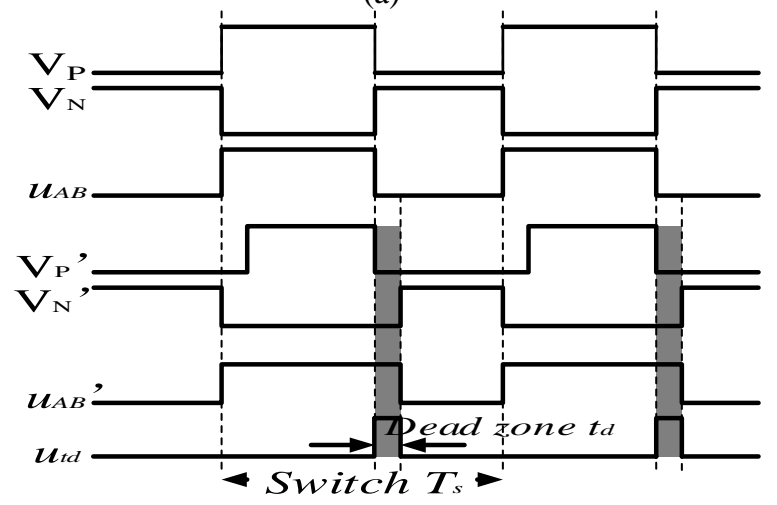

(b) 


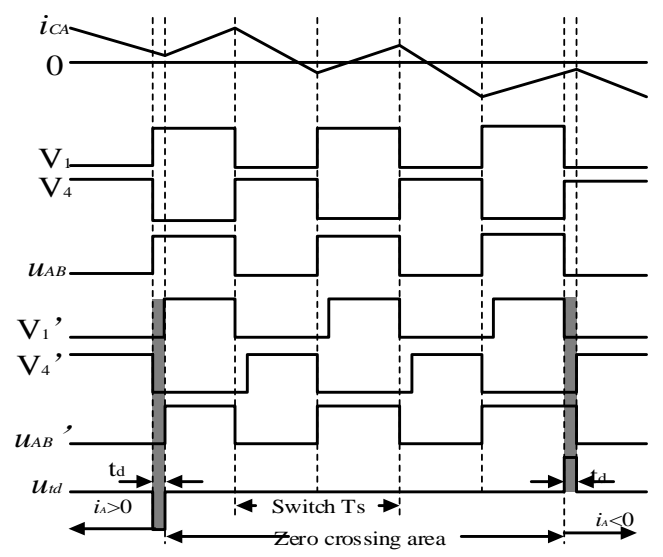

(c)

Figure 4. Output voltage dead time effect analysis: (a) $i_{C A}>0$ region, (b)

$$
i_{C A}<0 \text { region, (c) } i_{C A} \text { zero area. }
$$

To simplify the analysis, assume the following ideal conditions: (1) ignore the IGBT off time, conduction time, and conduction drop; (2) the APF condition and the main circuit of the IGBT maintain constant temperature; (3) zero current clamping phenomenon does not occur during the dead time, which does not consider the effects of zero current clamping phenomenon; (4) the error voltage is equidistant distribution caused by dead time effect; (5) the main circuit of the APF control signal carrier ratio is high enough, that is IGBT switching frequency $>>$ the frequency.

Under the above ideal conditions, this paper proposed the dead time effect of harmonics modeling method, the detection of harmonic is a stationary harmonic (such as $\mathrm{H}$ harmonic analysis), as shown in Figure 5. In the figure, $u_{C h}$ is the schematic diagram of $H$ harmonic voltage waveform of the main circuit of the APF output in a short period of $\mathrm{H}$ harmonics in $T_{h}$, assume in this harmonic period $T_{h}$, positive and negative time is equal. $i_{C h}$ and $\varphi$ respectively is output $h$ harmonic voltage main circuit of the APF corresponding to the $H$ current and the output harmonic compensation of $H$ harmonic power factor angle; $T_{Z}$ is $\mathrm{h}$ harmonic compensating current $i_{C h}$ zero zone time; $U_{t d m}$ is square wave amplitude of the average dead voltage $u_{t d}$ according to area equivalent principle.

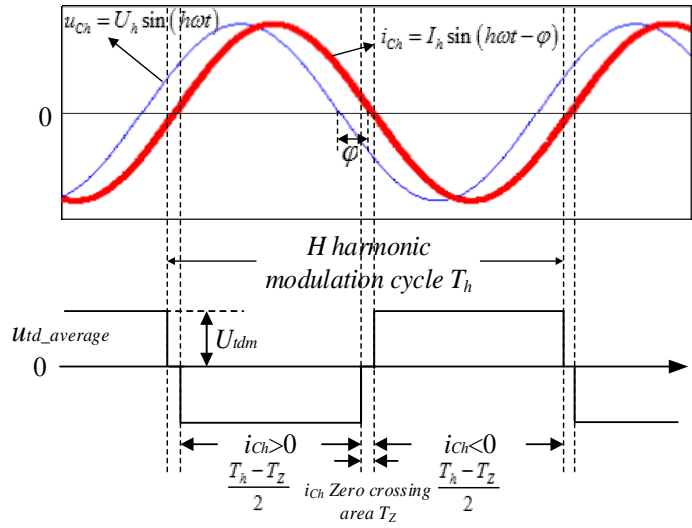

Figure 5. Schematic diagram of average dead voltage waveform during $h$ harmonic modulation period.

Assume that each error caused by dead time effect of the main circuit of APF is the same. Under the ideal assumptions (5), the compensation current is a random event if it belongs to area greater than zero or less than zero, that is to say the two random events are more occurred, according to the law of large numbers, in a certain period of time, the two random events occur with equal probability, then we have the hypothesis that in $T$ periods of a $h$ harmonic modulation, the number of dead time which the compensation current greater than zero is same with that less than zero, which is assumed to be $N$. According to the equivalent area approximation theory, in $h$ harmonic modulation period $T$, average dead time voltage waveform $u_{t d}$, namely $u_{t d}$ square wave amplitude can be expressed as:

$$
U_{t d m}=\frac{N t_{d}}{T} \times U_{d c}
$$

Therefore, the average dead time voltage $u_{t d \text {-average }}$ can be expressed by the formula:

$$
U_{\text {td_average }}=\left\{\begin{array}{cc}
u_{t d m} & i_{C h}<0 \\
0 & i_{C h}=0 \\
-u_{t d m} & i_{C h}>0
\end{array}\right.
$$

Furthermore, the average dead voltage $u_{t d \text {-average }}$ can be disassembled by Fourier decomposition: 


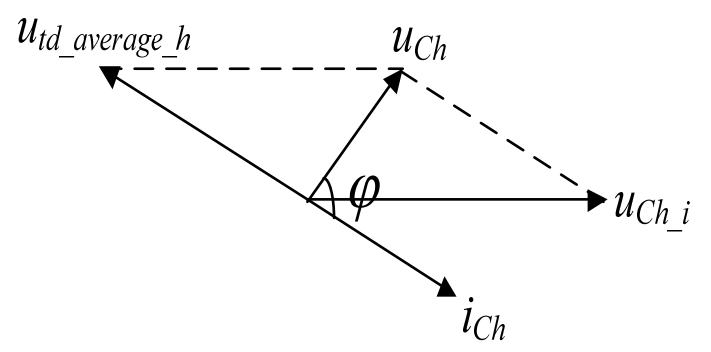

Figure 6. H harmonic vector relatioship.

$u_{t_{-} \text {average }}=\sum_{H=1}^{\infty}\left(-\frac{4 U_{t d m}}{H \pi} \cos H h \omega \frac{T_{Z}}{2}\right) \sin (H h \omega t-\varphi),(H=1,3,5, \cdots)$

By formulas (2) and (4), the Fourier series expressions

for the average dead voltage $u_{t d-\text { average }}$ can be:

$u_{t_{\text {_ }} \text { average }}=\sum_{H=1}^{\infty}\left(-\frac{4 U_{d c}}{H \pi} \frac{N t_{d}}{T_{h}} \cos H h \omega \frac{T_{Z}}{2}\right) \sin (H h \omega t-\varphi),(H=1,3,5, \cdots)$

Equation (5) shows the main circuit of the APF dead time effect of the voltage model under ideal condition considered zero regional effects, the model can get the following conclusion:

$$
u_{t d_{-} \text {average } h}=\left(-\frac{4 U_{d c}}{\pi} \frac{N t_{d}}{T_{h}} \cos h \omega \frac{T_{Z}}{2}\right) \sin (h \omega t-\varphi)
$$

From Eq. (6) we can see the dead time effect on the DC output voltage of APF $h$ harmonics influence and the main circuit voltage $U_{d c}$, the number of switches $N$ during $H$ harmonic modulation period, dead time $t_{d}, H$ compensation harmonic output power factor angle $\varphi$. The more the DC side voltage, switching frequency and dead time, the output voltage $H$ harmonic loss is bigger. Since the phase of dead voltage drop of $H$ components and the APF output h harmonic current is reversed, and the phase difference with APF output $h$ harmonic compensation voltage is angle $\varphi$, assume that ideal $h$ compensation harmonic voltage without dead zone is $u_{C h_{-} i}$, considering the dead zone, the vector relationship between the $H$ harmonics $u_{t d_{-} \text {average } h}$ of the average dead time voltage caused by the dead time effect ,

ideal $h$ compensating harmonic voltage $u_{C h_{-} i}$, the actual APF output $H$ compensation voltage $u_{C h}$, and APF output $h$ harmonic current of $i_{C h}$ vector is shown in Figure 6.

From Figure 6, when $\varphi=0$, namely $h$ harmonic output power factor $P F_{h}=1$, the phase of the $H$ harmonic voltage components brought by dead time effect and ideal compensation voltage $h$ harmonic component was reversed, at this time, the $H$ harmonic loss of output voltage caused by dead time effect become maximum; with the increase of phase difference $\varphi$, harmonic voltage loss caused by the dead zone effect decreases gradually.

(2) When $H \neq 1$, other harmonic components of $u_{t d-a v e r a g e H h}$ brought by dead time effect can be expressed as:
(1) When $H=1, \mathrm{H}$ harmonic components $u_{t d_{-} \text {average } \_} h$ dead time effect can be expressed as:

$$
u_{t_{-} \text {averageh } h}=\left(-\frac{4 U_{d c}}{H \pi} \frac{N t_{d}}{T_{h}} \cos \left(H h \omega \frac{T_{Z}}{2}\right)\right) \sin \left(H h \omega t-\varphi_{A}\right),(H=3,5,7, \cdots)
$$

From the above equation, the dead time effect of the output voltage of the APF contains a lot of $H(H=3,5,7, \ldots)$ Harmonic component. Because in the ideal condition, due to 
the symmetry of the dead voltage harmonic voltage, other components only containing $H(H=3,5,7, \ldots)$ odd times the sine term caused by dead time effect. Moreover, the greater the DC side voltage or the dead time is, the greater other harmonics caused by dead time effect, with the increasing number of harmonics, harmonic amplitude decreased.

From the above analysis, influence of dead time effect on the output characteristics of APF is reflected in the output voltage of the dead. By formula (4), the dead voltage is related to current zero crossing time of $T_{Z}$ length. Factors influencing the duration of zero current is mainly reflected in the following aspects, (1) the different control method of APF, make the current zero length different; (2) the main circuit of APF contains the inductance element in general, namely the inductor current ripple, which make the real APF output compensation harmonic current has certain width and need certain time to cross through the zero current point; (3) when the APF compensation harmonic current cross zero point in dead time, zero current clamping phenomenon may occur, which make the compensation current change rate fall, zero time compensation current delay. The higher the $T_{Z}$ is, the longer the current zero crossing time, and the smaller the APF output voltage harmonic loss; but with the increase of $T_{Z}$, may make the $H T_{Z}=T$ easy to appear, by formula (7), at this time, $H h$ harmonic components caused by the harmonic effect may actually increase.

Simulate the APF ideal dead time effect, when the presence of the dead, assuming the APF IGBT main circuit control dead zone $t d=3 \mu$ s. The dead zone makes the compensation current phase delay, after APF make harmonic compensation for nonlinear load, current waveform and spectrum analysis is shown in Figure 7(b). Although the compensated current waveform is improved, but the improvement effect slightly worse than the ideal effect without dead zone, then THDi=3.04\%. When considering other nonideal factors, it may further influence the dead time effect on APF compensation performance.

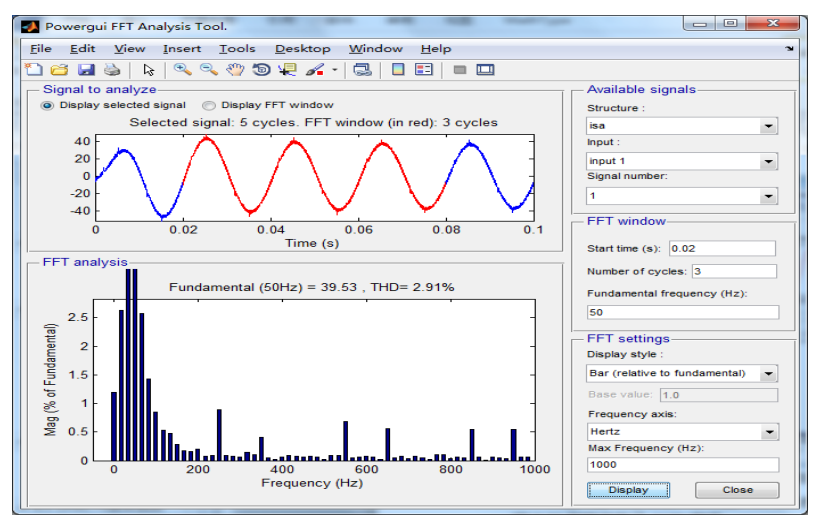

(a)

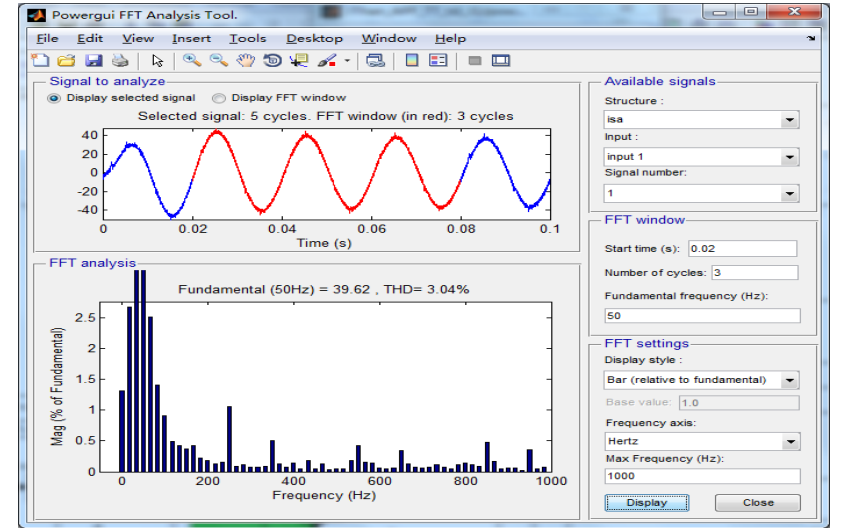

(b)

Figure 7. Has or no dead time compensation effect of APF. (a) No dead time compensation. (b) Dead time compensation.

\section{CONCLUSIONS AND FUTURE WORK}

This paper combines the theory derivation and simulation to analysis the negative effects of setting dead time of APF main control circuit. The analysis method of specific harmonic and the mechanism of control dead time effect on APF compensation performance are studied.

On this basis, some reasonable methods are finally put forward to reduce the dead time effect. From the above analysis, the APF dead time effect is mainly related to the main circuit DC voltage, switching frequency, dead time compensation current, power factor, and other factors. From Eq. (5), changing control strategy or control parameters of the main circuit switch number in theory can reduce the dead time effect. However, the reduction of switching frequency will increase the compensation error caused by the PWM modulation. So the control strategy parameters should be integrated into considering in practical application to make the APF compensation performance best. The required dead time of the different power switching devices is different, therefore, we can choose devices whose switch off time are shorter to reduce dead time, thus reducing the dead time effect. But any switching devices will be affected by many nonideal factors, which influence the off time, therefore the enough dead time need to add to ensure effective switch device fault.

The two above methods are only as auxiliary method to reduce the dead time effect. Considering the dead time effect is due to mainly dead time which brought a series of error square wave to APF output, so a series of corresponding with the dead zone of opposite polarity square wave can join to compensate the dead time effect in the APF output voltage compensation, it can effectively reduce the dead zone effect. But this method requires real-time judgment of compensation current direction, the real-time requirements are more high, and calculation is also more complicated.

\section{ACKNOWLEDGMENTS}

This work was supported by the National Natural Science Foundation of China No. 51177011, National High Technology Research and Development of China 
(863) No. 2012AA050210 and China State Grid Corporation project of science and technology.

\section{REFERENCES}

[1] Wang Zhaoan, Yang Jun, et al., The Harmonic Elimination and Reactive Power Compensation. Mechanical Industry Press, Beijing, 2010

[2] J.K. Phipps, J.P. Nelson, P.K. Sen, Power quality and harmonic distortion on distribution system. IEEE Trans. Ind. Appl., 30(2), pp. 476-483,1994.
[3] A.E. Emanuel, A. John, et al., A survey of harmonic voltages and currents at the customer's bus. IEEE Transactions of Power Delivery, 8(1), pp. 411-421, 1993.

[4] L. Morning, Cascaded multilevel dynamic voltage restorer compensating voltage drop. Master of technology degree thesis. Chongqing University School of electrical engineering, Chongqing, 2010.

[5] Y. Honggeng, X. Xianyong, L. Junyong, Progress in research and technology of power quality issues (six). Electric Power Automation Equipment, 24(3), pp. 1-5, 2004.

[6] L. Malesani, P. Mattavelii, et al., Robust dead-beat current control for PWM rectifier and active filters. IEEE Trans, 35(3), pp. 613-620, 1999.

[7] P. Mattavelli, A closed-loop selective harmonic compensation for active filters. IEEE Trans, 37(1), pp. 81-89, 2001. 